\title{
DIAGRAMAS DE INTERACCIÓN PARA SECCIONES METÁLICAS CIRCULARES A FLEXOTORSIÓN SIMPLE
}

\author{
(INTERACTION GRAPHS FOR STEEL CIRCULAR SECTIONS SUBJECTED TO SHEAR FORCE \\ WITH BENDING AND TWISTING)
}

Ramón Irles Más, Dr. ICCP, Prof. Tit. Univ.

$\mathrm{Dt}^{\circ}$. Ing. de la Construcción, OP e Inf. Urbana

Francisco Irles Más, ITOP, Prof. Tit. EU

Fecha de recepción: 21 - IX - 94

$\mathrm{Dt}^{\circ}$. Expresión Gráfica y Cartografía

422-26

Universidad de Alicante

Daniel Carratalá Climent, AT, Estudiante de $4 .^{\circ}$ curso Arquitectura

Universidad Politécnica de Valencia

ESPAÑA

\section{RESUMEN}

El uso de los diagramas de interacción como berramienta de predimensionado o dimensionado de secciones de piezas prismáticas sometidas a combinaciones de varios esfuerzos constituye algo habitual en la técnica. Desde hace tiempo se dispone de colecciones de estos diagramas para las diversas tecnologias: hormigón armado, acero estructural y secciones mixtas, bajo diversas solicitaciones.

En este articulo se expone minuciosamente el planteamiento para la obtención de los diagramas correspondientes a secciones metálicas circulares sometidas a la acción simultánea de esfuerzos cortante, flector y torsor. En particular, se realiza el estudio de la superficie limite de interacción de estos tres esfuerzos, en dichas secciones.

\section{SUMMARY}

Interaction graphs are extensively used as a tool for designing prismatic member sections subjected to several combined stresses. For a long time, sets of these graphs are available in building technologies: reinforced concrete, steel and composite sections, under various stresses.

This paper shows the formulation for obtainning the graphs corresponding to simultaneously bent and twist steel circular sections with shear force. In particular, a detailed analysis of the interaction limit surface is made for those stresses over the above cited sections.

\section{Introducción}

Este artículo desarrolla un tema dentro del mismo ámbito que el recientemente presentado por los mismos autores (5), y aporta el estudio y construcción de los diagramas de interacción para secciones circulares con cortante, flector y torsor. Hay que hacer referencia, por tanto, a las mismas publicaciones (1), (2), (3) y (4) alli citadas.
En el presente artículo se plantea una cuestión más particular, pero cuyo estudio ofrece la circunstancia especial de que la superficie de interacción está compuesta por dos regiones de diferentes características (una de ellas plana), correspondientes a distintas posiciones del punto más solicitado de la sección, que corresponden, a su vez, a distintos intervalos de preponderancia relativa de determinados esfuerzos de entre los considerados. 

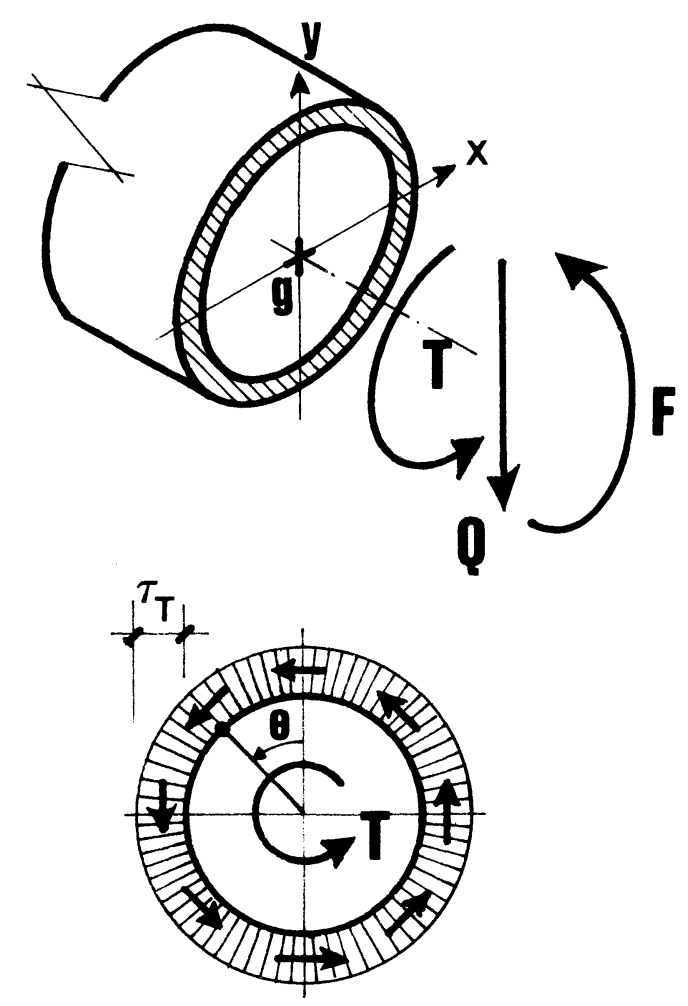

\section{Planteamiento del problema}

\subsection{Condición de agotamiento}

En la Fig. 1 se representan los esfuerzos considerados y las tensiones generadas en la sección circular.

La expresión analítica de las tensiones correspondientes es*:

$$
\begin{gathered}
\sigma_{\mathrm{F}}=\frac{\mathrm{F}}{\mathrm{I}_{\mathrm{F}}} \mathrm{R} \cos \theta=\frac{2 \mathrm{~F}}{\mathrm{I}_{\mathrm{T}}} \mathrm{R} \cos \theta=\frac{2 \mathrm{~F}}{\mathrm{~W}} \cos \theta \\
\tau_{\mathrm{T}}=\frac{\mathrm{T}}{\mathrm{I}_{\mathrm{T}}} \mathrm{R}=\frac{\mathrm{T}}{\mathrm{W}} \\
\tau_{\mathrm{Q}}=\frac{\mathrm{Q} \mathrm{M}_{\mathrm{EST}}}{\mathrm{tI}_{\mathrm{F}}}=2 \frac{\mathrm{QR}_{\mathrm{M}}^{2} \operatorname{sen} \theta}{\mathrm{RW}}
\end{gathered}
$$

siendo $\mathrm{W}=\mathrm{I}_{\mathrm{T}} / \mathrm{R}$ el módulo resistente a torsión; por tanto, la tensión de comparación en un punto gené-
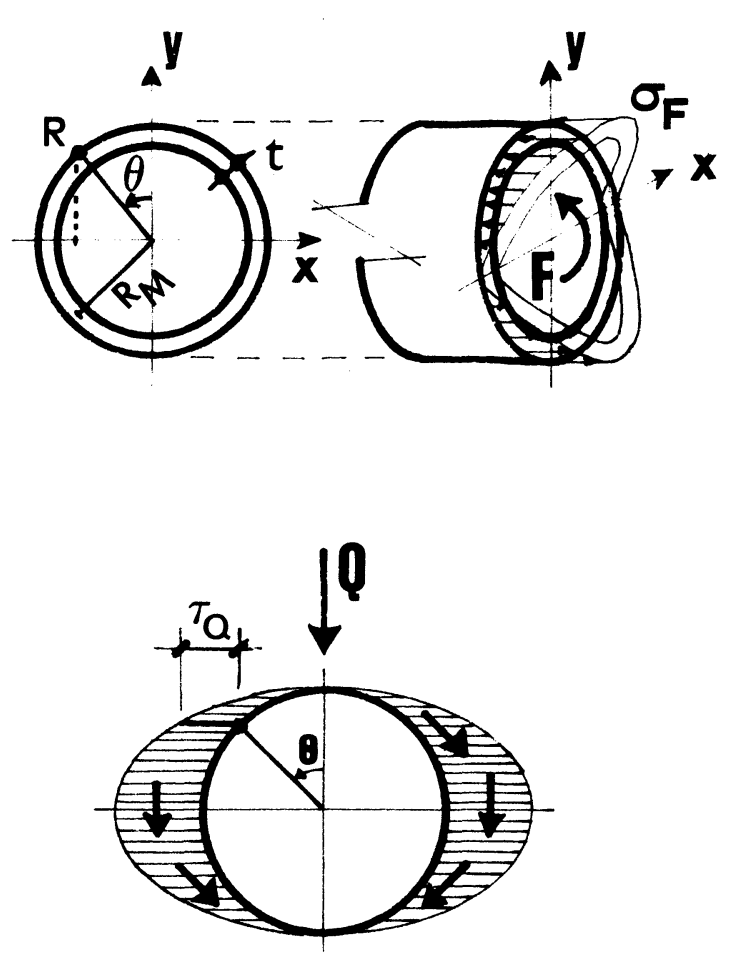

Fig. 1.

rico del contorno exterior, donde sin duda se encontrará su máximo valor, se expresa:

$\sigma_{\text {co }}=\sqrt{\sigma^{2}+3 \tau^{2}}=\sqrt{\left(\frac{2 \mathrm{~F} \cos \theta}{W}\right)^{2}+3\left(\frac{\mathrm{T}+2 \mathrm{QR}_{\mathrm{o}} \operatorname{sen} \theta}{\mathrm{W}}\right)^{2}}=\sqrt{\mathrm{f}(\boldsymbol{\theta})}$

donde $\mathrm{R}_{\mathrm{O}}=\mathrm{R}_{\mathrm{M}}^{2} / \mathrm{R}$,

en función de la única variable $\theta$ que define la posición del punto considerado.

Con el criterio de agotamiento habitual de que la tensión de comparación del punto que mayor la tenga alcance el límite elástico, la condición será:

$$
\sigma_{\mathrm{e}}=\sqrt{\mathrm{f}\left(\theta_{\max }\right)}
$$

o bien:

$$
\begin{gathered}
\sigma_{\mathrm{e}}^{2}=\mathrm{f}\left(\boldsymbol{\theta}_{\max }\right)=\left(4 \mathrm{~F}^{2} \cos ^{2} \theta_{\max }+3 \mathrm{~T}^{2}+12 \mathrm{TQR} \mathrm{R}_{\mathrm{o}} \operatorname{sen} \theta_{\max }+\right. \\
\left.+12 \mathrm{Q}^{2} \mathrm{R}_{\mathrm{o}}^{2} \operatorname{sen}^{2} \theta_{\max }\right) / \mathrm{W}^{2}
\end{gathered}
$$

\footnotetext{
* La expresión de $\tau_{\mathrm{Q}}$ utilizada es la habitual para perfiles de pared delgada, que facilita el promedio de $\tau$ a todo espesor en cada punto de la línea media de la misma; esta expresión pierde precisión cuando dicho espesor es grande, y validez para la sección maciza.
} 


\subsection{Búsqueda del punto más solicitado}

Como las funciones de tensiones [1] son continuas y con validez en todo el intervalo $\theta \boldsymbol{\epsilon}[0,2 \pi]$, el valor de $\theta_{\max }$ puede obtenerse con la condición matemática de máximo:

$$
\begin{aligned}
f^{\prime}(\theta)= & =\left(-8 F^{2} \operatorname{sen} \theta \cos \theta+12 T Q R_{o} \cos \theta+\right. \\
& \left.+24 Q^{2} R_{o}^{2} \operatorname{sen} \theta \cos \theta\right) / W^{2}
\end{aligned}
$$

ecuación que tiene las siguientes soluciones en el intervalo $\theta \in\left[0, \frac{\pi}{2}\right]^{*}$

$$
\text { a) } \cos \theta=0 \rightarrow \theta_{1}=\frac{\pi}{2}
$$

Esta solución existe siempre, cualesquiera que sean los valores de los esfuerzos ( $T, F, Q$ ), aunque no siempre se trata de un máximo, como veremos

b) $-8 \mathrm{~F}^{2} \operatorname{sen} \theta+12 \mathrm{TQR} \mathrm{R}_{\mathrm{o}}+24 \mathrm{Q}^{2} \mathrm{R}_{\mathrm{o}}^{2} \operatorname{sen} \theta=0 \rightarrow \theta_{2}=$

$$
=\operatorname{arcsen} \frac{3 T Q R_{O}}{2 F^{2}-6 Q^{2} R_{O}^{2}}
$$

Esta solución sólo es real cuando $\left[3 \mathrm{TQR}_{\mathrm{O}} /\left(2 \mathrm{~F}^{2}-\right.\right.$ $\left.\left.-6 \mathrm{Q}^{2} \mathrm{R}_{\mathrm{O}}^{2}\right)\right] \boldsymbol{\epsilon}[0,1]$

Es decir (restringiéndonos a valores positivos de $\mathrm{T}$, F, Q, habida cuenta de la simetría del problema), cuando:

$$
2 \mathrm{~F}^{2}-6 \mathrm{Q}^{2} \mathrm{R}_{\mathrm{o}}^{2}>0
$$

$$
3 T R_{o} \leq 2 F^{2}-6 Q^{2} R_{o}^{2} \rightarrow C_{1}(T, F, Q)=6 Q^{2} R_{o}^{2}+3 T Q R_{o}-2 F^{2} \leq 0 \quad[6 . b]
$$

simultáneamente.

Esta solución adopta dos valores particulares:

$\theta_{2}=\theta_{1}=\frac{\pi}{2}$, cuando [6.b] se cumple estrictamente $\left(C_{1}=0\right)$.

$\theta_{2}=0$, cuando se anula $\mathrm{T}$ ó $\mathrm{Q}$.

\subsection{Discusión de los valores máximos}

De ellas, los máximos relativos corresponden a los valores $(T, F, Q)$ que hacen estrictamente negativa la segunda derivada:

$f^{\prime \prime}(\theta)=\left[\left(24 Q^{2} R_{O}^{2}-8 F^{2}\right) \cos 2 \theta-12 T Q R_{o} \operatorname{sen} \theta\right] / W^{2}<0 \quad[7]$
Para cada caso, tendremos:

a) f' $(\pi / 2)=\left(8 F^{2}-24 Q^{2} R_{o}^{2}-12 T Q R_{o}\right) / W^{2}<0$

es decir, simplificando factores comunes:

$$
\mathrm{C}_{1}=6 \mathrm{Q}^{2} \mathrm{R}_{\mathrm{O}}^{2}+3 \mathrm{TQR}-2 \mathrm{~F}^{2}>0
$$

Sólo en esa región del espacio ( $\mathrm{T}, \mathrm{F}, \mathrm{Q})$ hay un máximo relativo en $\theta_{1}=\pi / 2$ (y fuera de ella un mínimo en el mismo punto, puesto que allí siempre se anula la $1 .{ }^{a}$ derivada; en la frontera $\mathrm{C}_{1}=0$, la función $\mathrm{f}(\boldsymbol{\theta})$ carece de curvatura, pero como es simétrica respecto de $\theta_{1}$, no presenta punto de inflexión, sino que será constante en $[0, \pi / 2])$. Cuando en $\theta_{1}=\pi / 2$ hay un máximo, la superficie de interacción [2] vendrá representada en dicho espacio por la ecuación:

$$
\mathrm{T}+2 \mathrm{QR} \mathrm{o}_{\mathrm{o}}-\mathrm{W} \sigma_{\mathrm{e}} / \sqrt{3}=0
$$

b) $\mathrm{f}^{\prime \prime}\left(\theta_{2}\right)=\left[\left(24 \mathrm{Q}^{2} \mathrm{R}_{\mathrm{O}}^{2}-8 \mathrm{~F}^{2}\right) \cos 2 \theta_{2}-12 \mathrm{TQR} \mathrm{o}_{\mathrm{o}} \operatorname{sen} \theta_{2}\right] / \mathrm{W}^{2}<0$

que, sustituyendo de [5] y tras algunas operaciones, queda:

$$
\left(\mathrm{F}^{2}-3 \mathrm{Q}^{2} \mathrm{R}_{\mathrm{O}}^{2}\right)\left[4\left(\mathrm{~F}^{2}-3 \mathrm{Q}^{2} \mathrm{R}_{\mathrm{o}}^{2}\right)^{2}-9 \mathrm{~T}^{2} \mathrm{Q}^{2} \mathrm{R}_{\mathrm{o}}^{2}\right]>0
$$

Sólo en la región del espacio (T,F,Q) que satisface [10] tendremos un máximo en $\theta_{2}$. En tal caso, la superficie de interacción vendrá representada por la ecuación resultante de sustituir [5] en [2], y que tras algunas operaciones resulta:

$$
\begin{gathered}
4 \mathrm{~F}^{6}-24 \mathrm{~F}^{4} \mathrm{Q}^{2} \mathrm{R}_{\mathrm{o}}^{2}+36 \mathrm{~F}^{2} \mathrm{Q}^{2} \mathrm{R}_{\mathrm{o}}^{2}-9 \mathrm{~T}^{2} \mathrm{Q}^{2} \mathrm{R}_{\mathrm{o}}^{2} \mathrm{~F}^{2}+3 \mathrm{~T}^{2} \mathrm{~F}^{4}- \\
-9 \sigma_{\mathrm{e}}^{2} \mathrm{~W}^{2} \mathrm{Q}^{4} \mathrm{R}_{\mathrm{o}}^{2}+6 \sigma_{\mathrm{e}}^{2} \mathrm{~W}^{2} \mathrm{~F}^{2} \mathrm{Q}^{2} \mathrm{R}_{\mathrm{o}}^{2}-\sigma_{\mathrm{e}}^{2} \mathrm{~W}^{2} \mathrm{~F}^{4}=0
\end{gathered}
$$

\section{Interpretaciôn geométrica y discusión completa del problema}

\subsection{Dominios correspondientes a cada situación de máximo}

Las regiones del espacio (T,F,Q) en las que la posición del máximo de $f(\theta)$ viene explicitada por [4] o [5], quedan delimitadas por las desigualdades [8] y [10], respectivamente.

A continuación, se describe la caracterización de las mismas, si bien no se expone su análisis detallado, que excede de la extensión razonable de este artículo.

\footnotetext{
* Nótese que bastará buscar el valor máximo en dicho intervalo, habida cuenta de los sentidos considerados para Q y T, y de la simetría del problema.
} 

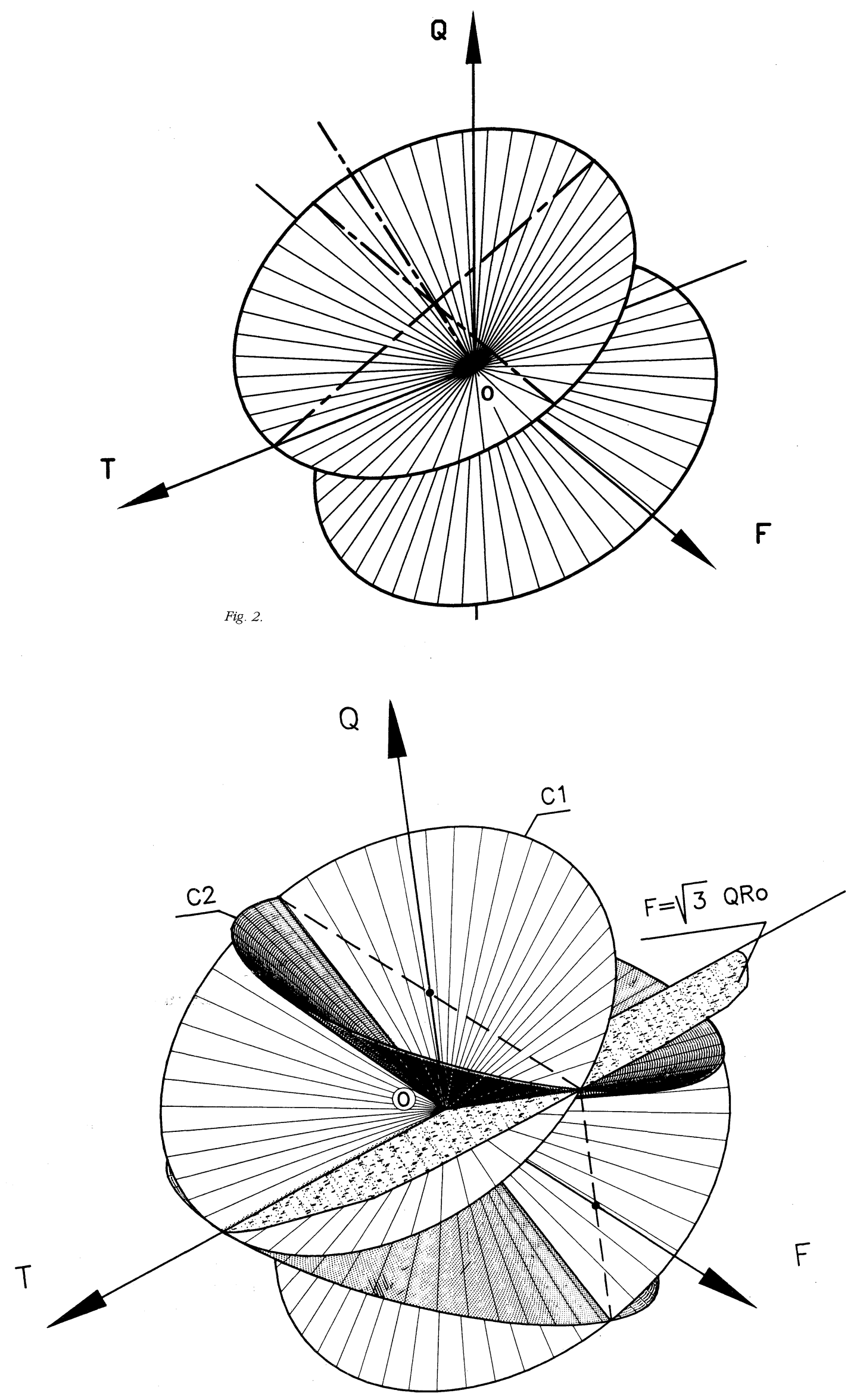

Fig. 3. 


\subsubsection{Máximo en $\theta_{1}=\pi / 2$}

La inecuación [8] la satisfacen los puntos (T,F,Q) interiores en dicho espacio a un cono $\mathrm{C}_{1}$ de directriz elíptica, vértice en el origen y eje contenido en el plano TQ, una de cuyas generatrices es el eje OT, que se representa en la Fig. 2.

En los puntos interiores a dicho cono, el máximo valor de $f(\theta)$ se produce en $\theta_{1}=\pi / 2$, mientras que fuera del cono, en el mismo punto, se presenta el mínimo valor de $f$.

\subsubsection{Máximo en $\theta_{2}=\operatorname{arcsen}\left[3 T Q R_{o} /\left(2 F^{2}-6 Q^{2} R_{o}^{2}\right)\right]$}

Puede demostrarse que la inecuación [10] se satisface:

a) En los puntos ( $T, F, Q)$ que estén simultáneamente:

- Fuera del cono $\mathrm{C}_{1}$.

- A la derecha del plano $\mathrm{F}=\sqrt{3} \mathrm{QR}_{\mathrm{O}}$ (Fig. 3).

b) En los puntos $(T, F, Q)$ que estén simultáneamente:
- Fuera del cono $\mathrm{C}_{2}$, simétrico del $\mathrm{C}_{1}$ respecto del plano FQ.

- A la izquierda del mismo plano $\mathrm{F}=\sqrt{3} \mathrm{QR}_{\mathrm{o}}$ (Fig. 3).

Si nos restringimos al $1{ }^{\text {er }}$ octante del espacio T,F,Q (sin restar generalidad al problema, dadas sus simetrías), todas estas condiciones se resumen en una sola: que la combinación $(T, F, Q)$ esté fuera del cono $C_{1}$. Esto es así porque en dicho octante la 2. ${ }^{2}$ condición de a) queda englobada en la primera, y la región que en el mismo cumple las b) queda dentro del cono $\mathrm{C}_{1}$, donde según [6.b] no es real la solución $\theta_{2}$ de la ecuación [3].

En resumen, puede decirse que en los puntos (T,F,Q) interiores al cono $C_{1}$ el máximo de $\mathrm{f}(\boldsymbol{\theta})$ se produce en $\theta_{1}$, y fuera del cono en $\theta_{2}$; justo sobre la superficie del mismo ocurre que $\theta_{2}=\theta_{1}$, y la variación de la posición del máximo es continua.

\subsection{Estudio de la superficie límite para máximo en $\theta_{1}$}

Cuando el máximo de $f(\theta)$ se produce en $\theta_{1}$, la superficie límite de interacción viene dada por [9].

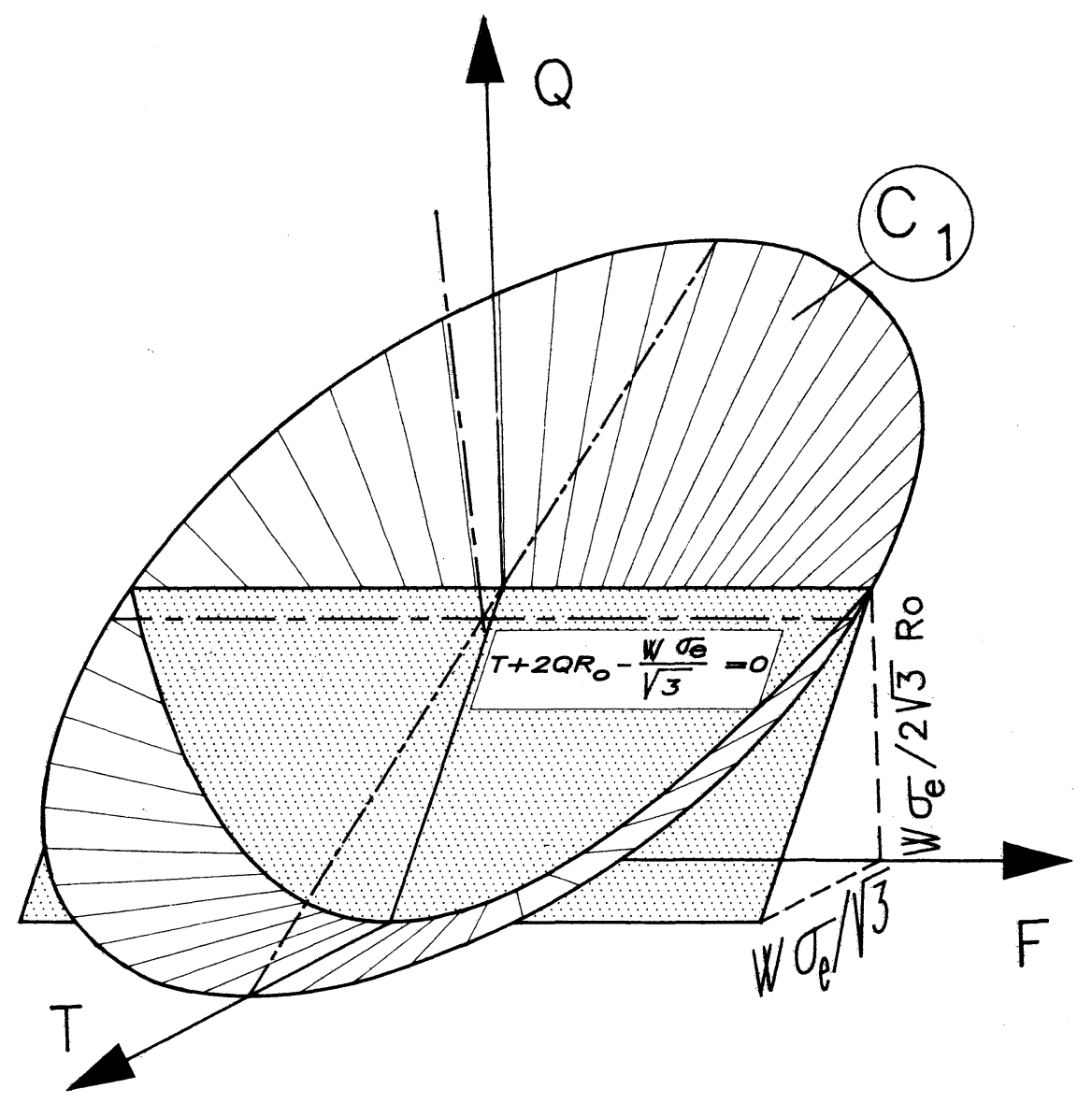

Fig. 4. 
Esta superficie es un plano en el espacio (T,F,Q) paralelo a la generatriz posterior del cono que, por tanto, $\mathrm{y}$ como se representa en la Fig. 4, corta al cono $\mathrm{C}_{1}$ en una parábola. Su dominio de validez se limita al interior del cono, es decir a la porción representada en la figura. Ello significa que en este dominio, el esfuerzo flector está desacoplado del resto.

\subsection{Estudio de la superficie limite para máximo en $\theta_{2}$}

Cuando el máximo de $f(\theta)$ se produce en $\theta_{2}$, la superficie límite de interacción viene dada por [11].

Esta superficie, bastante más compleja que la anterior, constituye la de interacción fuera del cono $\mathrm{C}_{1}$.
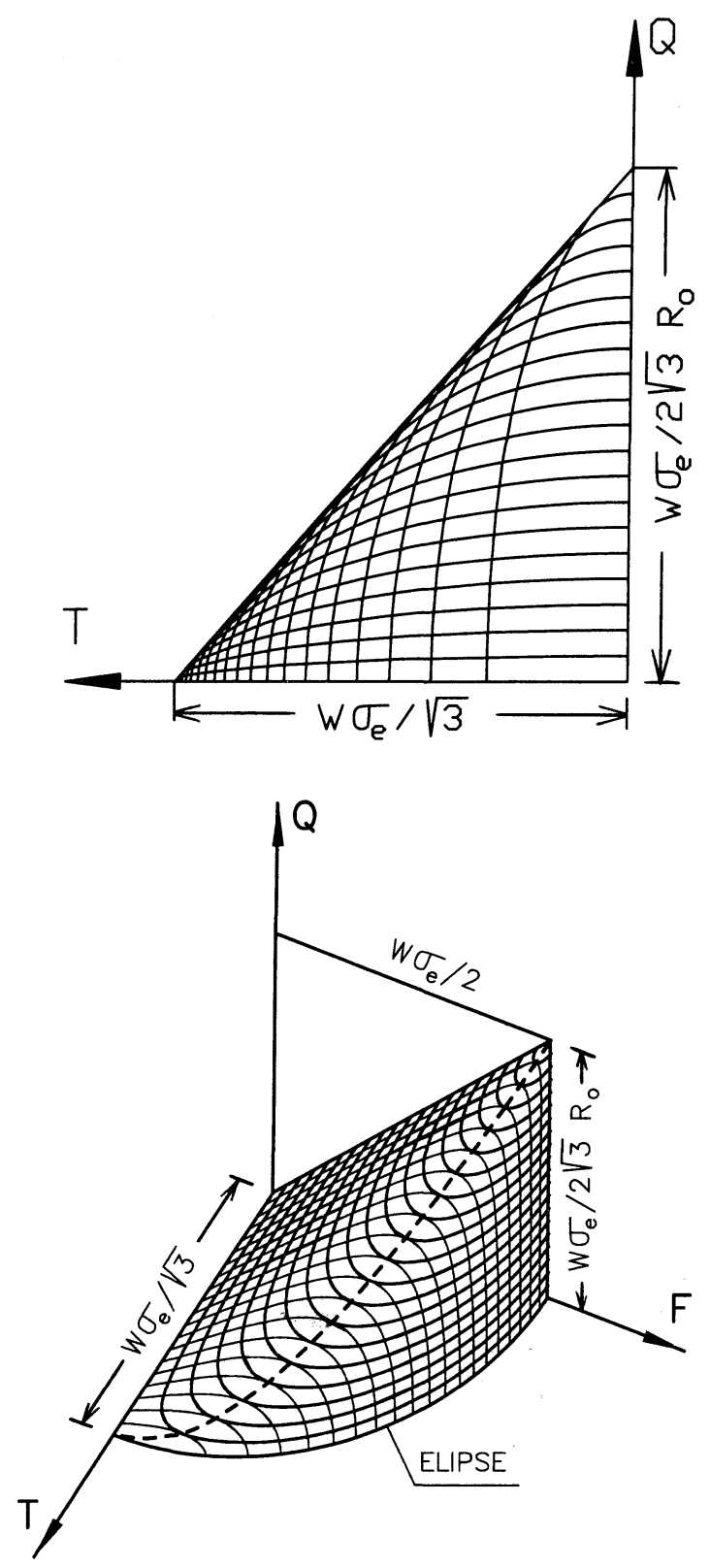

No puede pormenorizarse aquí su estudio detallado, pero sí algunas de sus conclusiones, que a continuación se exponen:

- La superficie [11] consta de tres hojas, dos de ellas planas. La tercera hoja presenta, en general, doble curvatura y consta, a su vez, de seis regiones: cuatro abiertas y no acotadas, y dos cerradas y acotadas, simétricas dos a dos respecto del plano TQ. En realidad, la superficie es simétrica respecto de los tres planos coordenados y pasa por el origen y por varias rectas.

- De esta superficie, sólo la porción de las regiones cerradas contenida en el 1 . $^{\text {er }}$ octante afecta al dominio del problema. En la Fig. 5 se representan sus
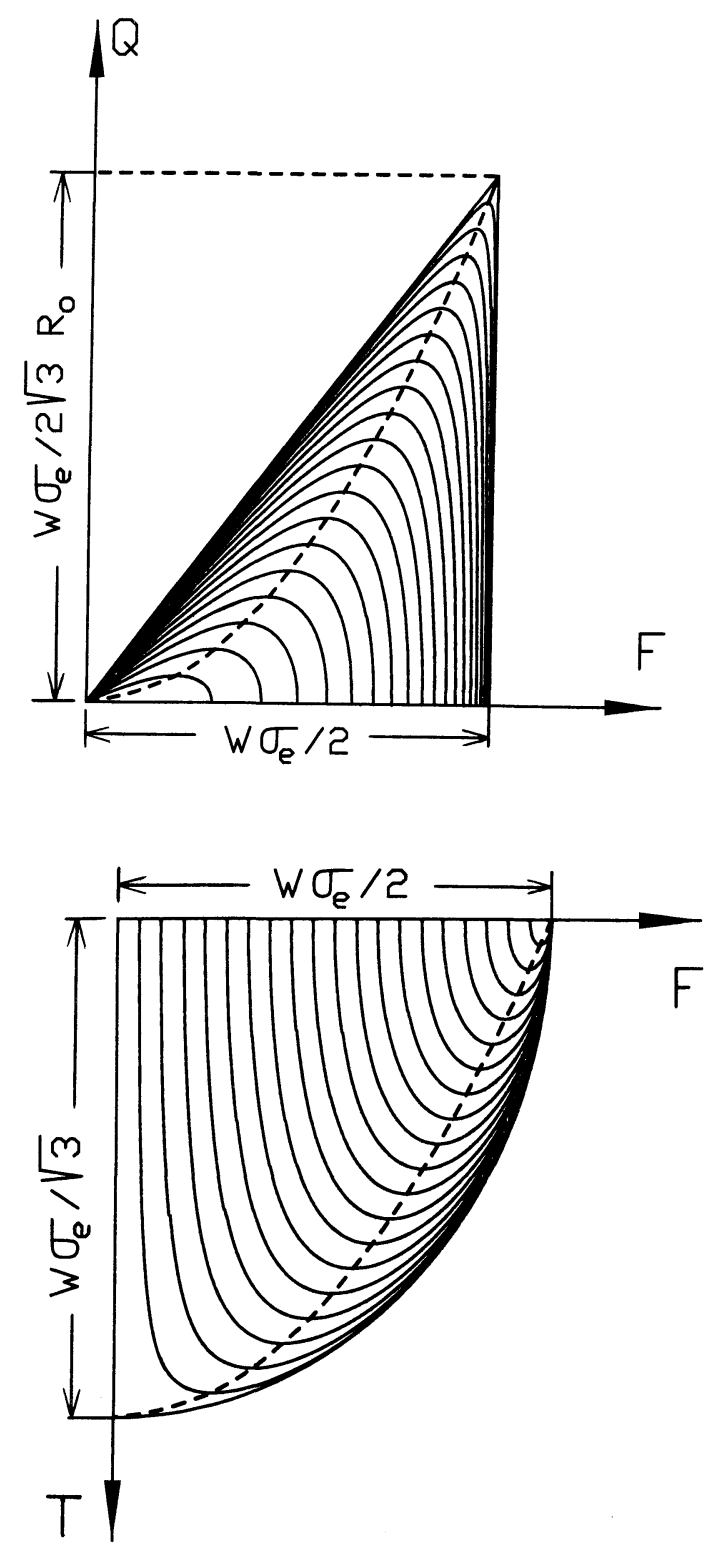
Informes de la Construcción, Vol. 46, n. ${ }^{\circ}$ 432, julio/agosto 1994

curvas de nivel según las tres proyecciones y una perspectiva, lo cual permite hacerse una idea de su forma.

- El plano dado por [9], que constituye la superficie de interacción en el caso de máximo en $\theta_{1}=\pi / 2$, es tangente a esta superficie a lo largo de una parábola que coincide con la intersección de dicho plano con el cono $\mathrm{C}_{1}$.

- Las regiones cerradas de la superficie están contenidas en un cilindro de eje vertical Q, cuya directriz es la elipse intersección de aquélla con el plano $Q=0$, y a lo largo de la cual la superficie y el cilindro son tangentes.

Por tanto, su dominio de validez para el problema está constituido por su porción en $1{ }^{\text {er }}$ octante, exterior al cono $\mathrm{C}_{1}$, al cual corta también según la citada parábola.

\section{Representación de la superficie limite. Diagramas de interacción}

Constituida la superficie límite por dos regiones, una de ellas plana y la otra alabeada, pero con continuidad de valor y de primeras derivadas en todas direcciones a lo largo de la parábola común, todos los diagramas de interacción posibles, representando sus líneas de nivel según planos paralelos a los coordenados, estarán constituidos por trazos mixtilíneos compuestos por un tramo recto y otro curvo, este último de diferente naturaleza según las secciones consideradas, y con continuidad de valor y de primera derivada en el punto de confluencia.

En la Fig. 6 se indica la generación de los tres posibles diagramas para las distintas secciones indicadas. Los correspondientes a secciones $\mathrm{F}=$ cte están constituidos por tramos rectos y elípticos, mientras que en los otros dos $(\mathrm{T}=$ cte o $\mathrm{Q}=$ cte), el tramo curvo viene representado por ecuaciones de grado 4, más complejas, pero con una de las variables explicitable en ambos.

En la Fig. 7 se representan los tres posibles diagramas de interacción para un perfil $\varnothing 100$ x 6 supuesto de un acero A42, preparados para su uso.
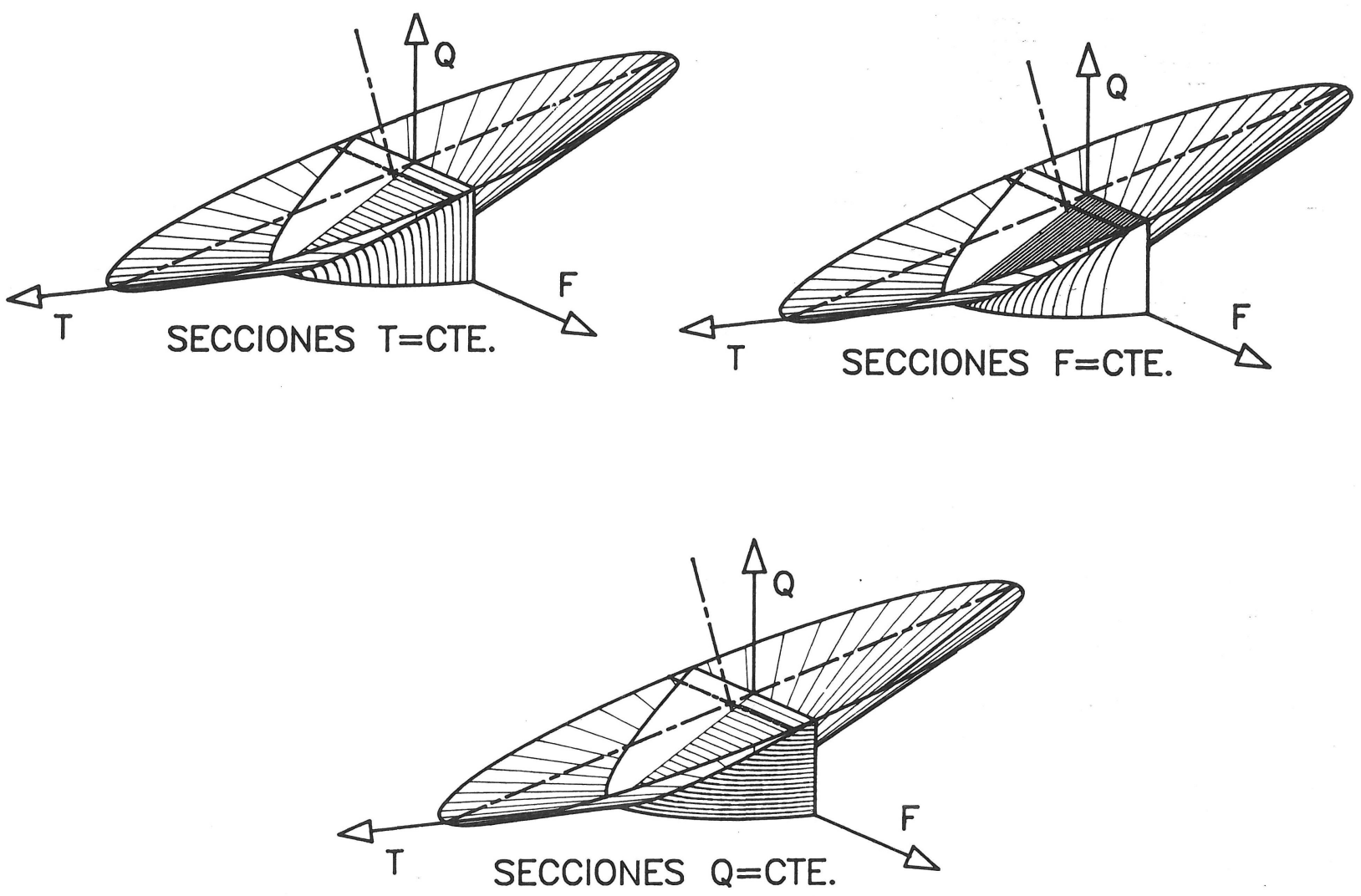

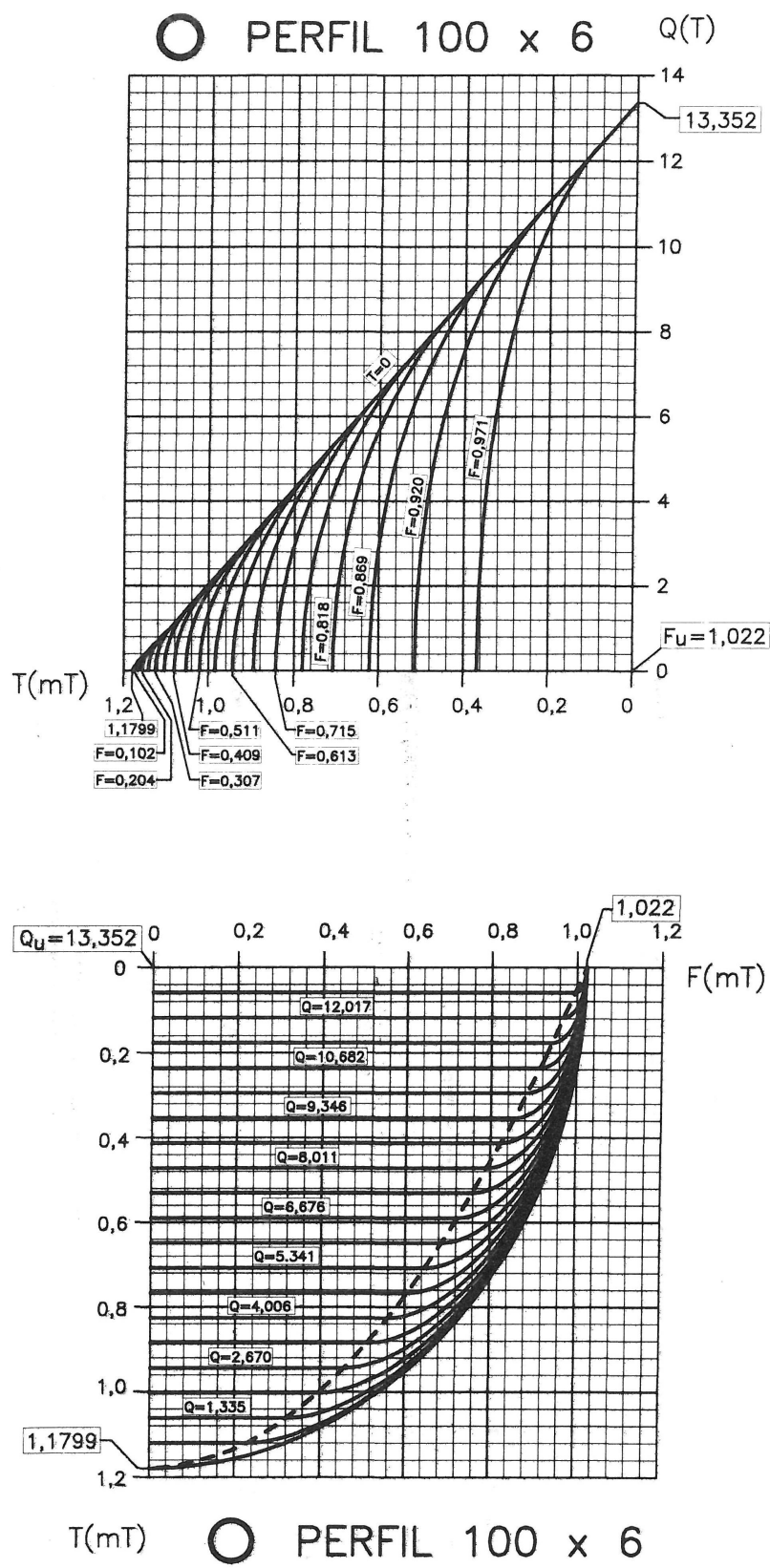

\section{Conclusión}

En este artículo se ha desarrollado la formulación analítica para la obtención de la superficie límite de interacción de los esfuerzos de corte, flexión y torsión, en los perfiles metálicos huecos circulares.

La superficie está constituida, en el correspondiente espacio $(T, F, Q)$, por una parte plana y otra alabeada.

Ha quedado expuesta la discusión completa del problema, que puede realizarse íntegramente mediante técnicas analíticas, con mayor o menor complejidad, según las características de cada región de la superficie.

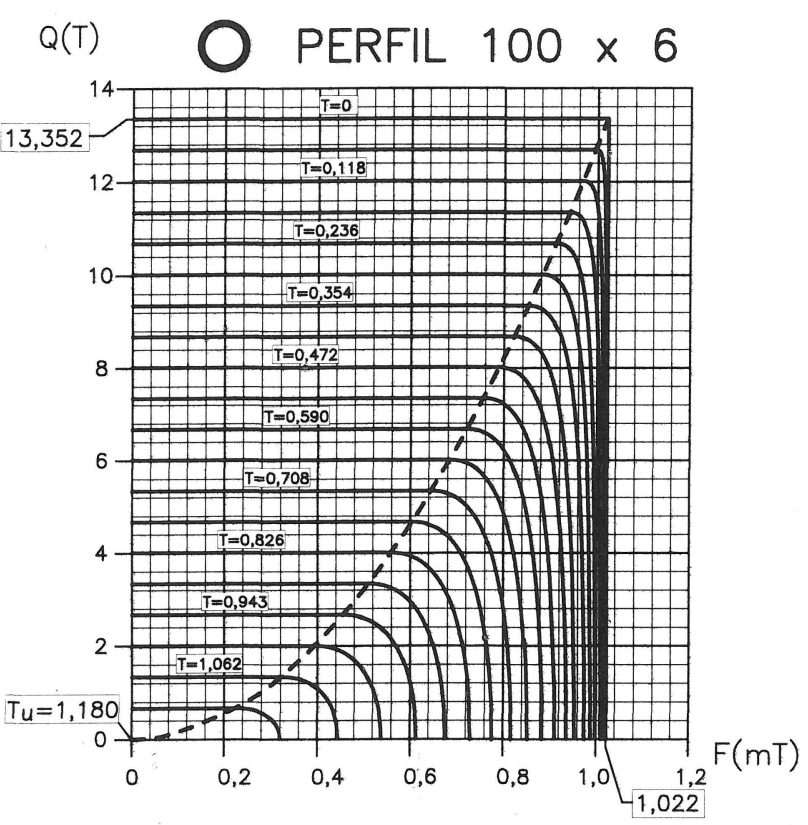

FIg. 7.

La superficie pone de manifiesto que, salvo para pares de torsión y flexión $(\mathrm{T}, \mathrm{F})$ próximos a sus valores máximos, esta última está desacoplada de los otros dos esfuerzos.

Como ejemplo, se han representado los tres posibles diagramas para un perfil $\varnothing 100 \times 6$.

\section{BIBLIOGRAFIA}

(1) P. J. Montoya, A. G. Meseguer y F. Morán: "Hormigón Armado", Gustavo Gili, Barcelona. Ed. 13, 1991.

(2) S. P. Zhou y W. F. Chen: "Design Criteria For Box Columns under Biaxial Loading", , Journal of structural Engrg., volumen 111, número 12, diciembre 1985.

(3) Atsuta, T., y Chen, W. F.: "Theory of Beam-Columns", tomo 2.', McGraw Hill B.C., 1976.

(4) García García, J., y López Pellicer Marfil, M.: "Álgebra lineal y Geometría", Alcoy, 4. ${ }^{a}$ ed., 1984.

(5) Irles, R.; Irles, F., y Carratalá, D.: "Diagramas de interacción para secciones metálicas huecas a flexión esviada compuesta", Informes de la Construcción, número 431, 1994. 